\title{
SEX RATIO EVOLUTION WHEN FITNESS VARIES
}

\author{
J. J. BULL \\ Laboratory of Genetics, University of Wisconsin, Madison, WI 53706 and School of \\ Biological Sciences, University of Sussex, Falmer, Brighton, Sussex, BN1 $90 G$ \\ Received 31.iii.80
}

\section{SUMMARY}

The sex ratio favoured by natural selection is studied in a single-locus model of a random-mating population. The population inhabits a "patchy" (spatially heterogeneous) environment, and offspring reared in different patches have corresponding differences in fitness, depending on their sex. Thus, some patches produce the best males; these or other patches produce the best females. The mating pool is comprised of the individuals from all patches. Sex ratio is allowed to evolve so that a zygote's probability of becoming male or female is determined in response to the patch in which it is reared. Results include the following:

(1) For many conditions, the sex ratio at equilibrium is one in which only males develop in patches relatively beneficial to maleness and/or only females develop in patches relatively beneficial to femaleness; the favoured sex ratio in some patches may include both sexes. brium.

(2) The average population sex ratio in zygotes is not generally $\frac{1}{2}$ at equili-

(3) If the frequency of patch types fluctuates between generations, there is a greater tendency for selection to favour a sex ratio of $\frac{1}{2}$ in each patch type than if the environment is temporally constant. This may help explain why so few animals control sex ratio in response to environmental cues.

(4) The sex ratio may coevolve with the offspring's choice of patch type and the environmental cue it uses in determining sex; when possible, embryos are selected to avoid patches detrimental to both sexes, and are selected to determine sex in response to the cue which indicates the greatest differential benefit to maleness versus femaleness. Available sex ratio data from haplo-diploids and invertebrates with environmental sex determination lend qualitative support to some of the predictions, but the widespread occurrence of environmental sex determination in reptiles offers a possible contradiction.

\section{INTRODUCTION}

SPECIES with separate sexes tend to produce males and females in equal numbers. Fisher (1930) first suggested a plausible evolutionary explanation, although the first algebraic presentation is by Shaw and Mohler (1953). Their argument is as follows; suppose that among zygotes, females are more common than males, then on average, each male zygote will transmit more genes to offspring than the average female, and this will be true despite subsequent differential mortality, monogamy, or polygyny. If some parents have a heritable tendency to produce more sons than average, thereby producing correspondingly fewer daughters, this son-producing tendency increases in frequency because the bias of sons in the brood transmits more genes than would have been transmitted if they had been daughters. The same argument applies to selection for daughters if there is a male surplus, 
and the equilibrium is at one son to one daughter in the population. Once this population equilibrium is reached there is no selection on the family sex ratio; parents producing only sons, only daughters, or a ratio of $\frac{1}{2}$, all have the same number of genes in grandchildren. (Sex ratio here and below refers to the proportion of males either at conception or at the time of sex determination.)

Shaw (1958) showed that Fisher's result does not apply to sex-linked inheritance of genes which modify sex ratio in the heterogametic sex, and Hamilton (1967) further delimited the assumptions behind Fisher's argument. Hamilton showed that Fisher's result is invalid if brothers tend to compete with each other for the same females. For example, if brothers mate only with their sisters and if one male can fertilize many females, then a mother leaves the most grandchildren by producing one son with the remainder as daughters. In this case, one son fertilizes the same number of females as, say, 10 sons combined, whereas one daughter produces only a tenth of the number of grandchildren as 10 daughters. (In the Fisher case, one son fertilizes a tenth the number of females as 10 sons.) Here, selection is on the family sex ratio.

Trivers and Willard (1973) introduced the idea that offspring fitness may vary with sex in association with special environmental circumstances. If a parent can produce either a daughter of average fitness or a son of aboveaverage fitness, then selection favours producing the son (provided the population sex ratio averages near $\frac{1}{2}$ ). Here selection operates on the sex ratio associated with a particular environmental circumstance, in the context of the population sex ratio. Trivers and Willard applied their model to sex ratios in mammals, but the same idea has been extended to hermaphroditic shrimp (Charnov et al., 1978), parasitic wasps (Charnov, 1979; Charnov et al., in preparation), and to species with environmental sex determination (Charnov and Bull, 1977). Charnov and Bull (1977) used the argument further, to explain the evolution of a genetic system: if selection favors becoming male (or female) under particular circumstances, then selection might thereby favour a genetic system which permits an individual to do this. Thus, environmental sex determination may be favoured over genotypic sex determination because it enables the embryo to control its sex in response to the environment.

The purpose of this paper is to provide an algebraic treatment of sex ratio evolution when offspring fitness varies as a function of environment circumstance. A chief motivation is to understand the evolution of environmental sex determination, a problem of special interest now that it has been shown to be widespread in reptiles and without obvious adaptive benefit (Bull, 1980). The models strictly apply to environmental sex determination, by assuming that the zygote controls sex determination, but it is shown that many of the conclusions apply regardless of whether the parent or offspring controls sex determination. This study follows two others on the same subject (Charnov, 1979; Charnov et al., 1978), providing a more rigorous basis for some earlier conclusions as well as presenting several new results.

\section{OfFSPRING FITNESS AS A FUNCTION OF ITS SEX: BIOLOGICAL EXAMPLES}

As a basis for the models given below various examples will be given in which the same underlying features are present. 
(a) (from Christie, 1929; Charnov and Bull, 1977). Some mermithid nematodes begin life as eggs in the soil or on vegetation. Soon thereafter the egg is ingested by an insect and the worm enters the insect's abdomen. As the worm develops, the insect's body contents are exhausted, and the worm emerges to become a free-living adult in the soil. Not all insects are alike, however. Some are large and others small, and they have differing levels of infestation. In general, the more host nutrient that the worm consumes, the larger its adult size. Large size may enhance adult fitness more for females than males.

(b) (from Charnov, 1979; Charnov et al. (in preparation). Some species of parasitic wasps lay one egg per host, and the wasp larva devours the host before pupating. Host size varies, either because different species are attacked or because hosts of different ages are attacked, and the size of the emerging parasite is again correlated with host size. Some other wasps nest in crevices, and crevice size varies throughout the environment. In these, offspring size is limited by the size of the hole in which the eggs are laid, because the offspring develops into an adult within the hole. Possibly, large size is more beneficial to female than male wasps.

(c) (from Trivers and Willard, 1973). In mammals, an individual's size as an adult may be reflected by its size at weaning. Fitness may again depend upon size, and in polygynous species, large size may enhance male fitness more than female fitness. If mothers differ in their ability to produce large offspring, then there will be corresponding differences in the fitnesses of these offspring upon reaching adulthood.

These examples have four properties in common.

(1) Offspring are raised in an environment which has a large effect on their fitness as an adult (the host, the crevice, the mother's care).

(2) The environment is spatially heterogeneous, so that offspring reared in some "patches" have higher fitness than offspring from other patches.

(3) Male and female offspring may differentially benefit from the quality of the patch.

(4) The mating pool of adults is likely to contain individuals from a wide array of patches.

To a greater or lesser extent, these properties may apply to many organisms. Populations are invariably exposed to moisture, thermal, or chemical gradients, and these may have lasting effects on offspring vigour. The models below will incorporate these four properties in attempting to understand how they affect sex ratio evolution. The question considered is: What sex ratio is favoured in each type of "patch" in the environment? To simplify the algebra, it will be assumed that the zygote or embryo controls its sex determination. Therefore the dynamic properties of the models apply only to environmental sex determination, because genotypic sex determination fixes the zygote's sex at or before conception. However, it will be shown that the equilibrium conditions are the same, regardless of whether a parent or offspring controls the sex ratio. Thus, many of the conclusions apply to species with genotpyic sex determination, provided genetic variation for family sex ratio exists among the parents.

\section{A SELECTION MODEL}

Diploid zygotes are distributed throughout an environment and settle, whereupon they undergo sex differentiation and grow into adults. 
Subsequently they mix, mate, and produce zygotes which are again distributed throughout the environment. The environment is spatially heterogeneous, consisting of patches. A patch type is defined by its effect on the fitness of zygotes reared within it. The adult fitness (viability times fertility) of a male and female reared in patch type $i$ is $W_{i}^{m}$ and $W_{i}^{f}$, respectively. (These and most other parameters are also listed in the Appendix.) Zygotes encounter patch type $i$ with frequency $k_{i}$, where $\sum_{i} k_{i}=1$. Sex is determined by the individual's genotype in response to patch type.

Analysis of selection is restricted to a single locus with 2 alleles having different effects on the sex ratio. In patch type $i$, the genotype $A A$ becomes male with probability $r_{i}^{* *}, A a$ with probability $r_{i}^{*}$, and $a a$ with probability $r_{i}$. The population is assumed infinite, zygotes are distributed into patches irrespective of genotype, and mating is random among individuals from all patch types. Assign each genotype an initial frequency in zygotes: $p_{11}$ for $A A, 2 p_{12}$ for $A a$, and $p_{22}$ for $a a$. The zygotic frequency of $A$ is $p_{11}+p_{12} \equiv p$. The objective is to determine the change in the frequency of $A$ due to selection on the sex ratio. We first calculate the expected frequency of $A$ among mating males (call it $x$ ) and among females $(y)$. Since $p_{11}^{\prime}$ is $x y$ and $p_{12}^{\prime}$ is $[x(1-y)+y(1-x)] / 2$, then $p^{\prime}=(x+y) / 2$. Comparing $p^{\prime}$ to $p$ determines the effect of selection on gene frequency. This is fundamentally the method of Wright and Dobzhansky (1946) and Wright (1969, p. 50), except that here we let genotypes influence fitness through the sex ratio, rather than directly affecting the $W$ 's.

The frequency of $A$ among males is calculated in the following way. Absolute numbers of individuals are ignored because only relative parameters are important in the final equations. Patch type $i$ contributes $k_{i}\left(p_{11} r_{i}^{* *}+2 p_{12} r_{i}^{*}+p_{22} r_{i}\right) W_{i}^{m}=k_{i} \bar{r}_{i} W_{i}^{m}$ males to the mating pool, where $\bar{r}_{i} \equiv p_{11} r_{i}^{* *}+2 p_{12} r_{i}^{*}+p_{22} r_{i}$, and males are counted after fitness effects. In the total pool, therefore, there are $\sum_{i} k_{i} \bar{r}_{i} W_{i}^{m}$ males. Of these, $\sum_{i} k_{i}\left(p_{11} r_{i}^{* *}+\right.$ $\left.p_{12} r_{i}^{*}\right) W_{i}^{m}$ contribute $A$. Therefore, the frequency of $A$ among mating males is

$$
\frac{\sum_{i} k_{i}\left(p_{11} r_{i}^{* *}+p_{12} r_{i}^{*}\right) W_{i}^{m}}{\sum_{i} k_{i} \bar{r}_{i} W_{i}^{m}} .
$$

Similarly, for females it is

$$
\frac{\sum_{i} k_{i}\left\{p_{\mathrm{n} 1}\left(1-r_{i}^{* *}\right)+p_{12}\left(1-r_{i}^{*}\right)\right\} W_{i}^{f}}{\sum_{i} k_{i}\left(1-\bar{r}_{i}\right) W_{i}^{f}} .
$$

These expressions may be condensed with the following notation,

$$
\bar{W}_{m}=\sum_{i} k_{i} \bar{r}_{i} W_{i}^{m} \quad \bar{W}_{f}=\sum_{i} k_{i}\left(1-\bar{r}_{i}\right) W_{i}^{f}
$$

$$
\begin{aligned}
& p \stackrel{*}{W}_{m}=\sum_{i} k_{i}\left(p_{11} r_{i}^{* *}+p_{12} r_{i}^{*}\right) W_{i}^{m} \\
& p \stackrel{*}{W}_{f}=\sum_{i} k_{i}\left[p_{11}\left(1-r_{i}^{* *}\right)+p_{12}\left(1-r_{i}^{*}\right)\right] W_{i}^{f},
\end{aligned}
$$


and we have

$$
p^{\prime}=p\left(\frac{\mathscr{W}_{m}}{2 \bar{W}_{m}}+\frac{\stackrel{*}{W}_{f}}{2 \bar{W}_{f}}\right)
$$

or

$$
\Delta p=p\left(\frac{\stackrel{*}{W}_{m}-\bar{W}_{m}}{2 \bar{W}_{m}}+\frac{\stackrel{*}{W}_{f}-\bar{W}_{f}}{2 \bar{W}_{f}}\right) .
$$

$\bar{W}_{m}$ is the population mean of male fitness times patch sex ratio; $\bar{W}_{f}$ is the corresponding term for females. $\stackrel{*}{W}_{m}$ may be thought of as a marginal fitness of allele $A$ in males; it is an average over the $A A$ and half of the $A a$ genotypes of male fitness times patch sex ratio (and accordingly, for ${ }^{*} W f$ ). Since (2) incorporates genotype as well as allele frequencies, it is not generally legitimate to iterate (2) repeatedly if only allele frequencies are known (this is dealt with below). Even so, (2) suggests a general interpretation of sex ratio evolution: selection acts on a modifier of sex ratio based on its effect in males relative to the mean product of male fitness times sex ratio, plus its effect in females relative to the mean product of female fitness times proportion of females. Since sex ratio modification usually involves increases through one sex and decreases through the other, the two effects are of opposite sign but not necessarily of equal magnitude. It may be noted that if $\stackrel{*}{W}_{m}$ and $W_{f}$ allows terms other than just sex ratio to differ among genotypes, (2) applies to a wide class of two-sex problems (Wright and Dobzhansky, 1946; Wright, 1969; Kidwell et al., 1977).

Although (2) has been derived for zygote control of sex ratio, a similar expression obtains if the maternal genotype controls sex rato,

$$
\left(\frac{x+y}{2}\right)=\frac{y}{2}\left(\frac{\stackrel{*}{W}_{m}-\bar{W}_{m}}{2 \bar{W}_{m}}+\frac{\stackrel{*}{W}_{f}-\bar{W}_{f}}{2 \bar{W}_{f}}\right) .
$$

The expression inside the right-hand parentheses may be written in terms similar to those in (1), but the genotype frequencies and sex ratios have different meanings. Here, genotype frequencies are measured among the adults after fitness effects, $x$ corresponding to $A$ among males and $y$ to $A$ among females. $\stackrel{*}{W}_{m}$ is then the average proportion of sons times fitness produced by $A A$ and half the $A a$ mothers,

$$
\frac{1}{y} \sum_{i} k_{i}\left(y_{11} r_{i}^{* *}+y_{12} r_{i}^{*}\right) W_{i}^{m},
$$

where $r_{i}^{* *}\left(r_{i}^{*}\right)$ refers to the proportion of sons of $A A(A a)$ mothers in patch type $i$, and so forth. From (2c) it is seen that the conditions for gene frequency equilibrium are exactly those of (2b). At equilibrium, therefore, one expects to observe the same fraction of males in patches of type $i$, regardless of whether a parent or the offspring controls sex ratio. Note that the rate of gene frequency change is less in (2c) than in (2b), due to the expression of allele $A$ being limited to one sex in (2c). If $\Delta(x+y) / 2$ is replaced by $\Delta(x / 2+y) / 2$, then (2c) applies to haplo-diploidy. 
Charnov (1979) studied a wide variety of sex ratio problems (including sex allocation in hermaphrodites) with a single model. He proposed that the evolutionarily stable equilibrium requires

$$
\frac{\stackrel{*}{W}_{m}}{2 \bar{W}_{m}}+\frac{\stackrel{*}{W}_{f}}{2 \bar{W}_{f}} \leqq 1,
$$

where he assumes that allele $A$ is so rare that second and higher order terms of its frequency can be neglected. Therefore, he obtains an equilibrium which cannot be invaded by rare modifiers of sex ratio. The above model (2) is therefore more general, in allowing one to consider common as well as rare alleles and in measuring the strength of selection. As a rule, however, the equilibrium predicted by Charnov's model is the same as that here.

Expression (2b) is readily simplified when $A$ has an additive effect on sex ratio in various patch types: $r_{i}^{* *}=r_{i}+2 \delta_{i}, r_{i}^{*}=r_{i}+\delta_{i}$. Expression (2b) becomes

$$
\Delta p=\frac{p}{2}\left(1+\frac{p_{11}}{p}-2 p\right)\left\{\sum_{i}\left[\frac{k_{i} \delta_{i} W_{i}^{m}}{\bar{W}_{m}}-\frac{k_{i} \delta_{i} W_{i}^{f}}{\bar{W}_{f}}\right]\right\} .
$$

In modifying this further, we rewrite $\bar{W}_{m}=\sum_{i} k_{i} r_{i} W_{i}^{m}+2 p \sum_{i} k_{i} \delta_{i} W_{i}^{m}$, and rewrite $\mathscr{W}_{f}$ similarly. The first term in this expression is the population mean of patch sex ratio times male fitness in the absence of allele $A$ ( $a$ fixed), and the second term is the contribution of $A$ to the population sex ratio. Thus, the denominator may be interpreted as the sum of a "residual" male fitness plus the effect of allele $A$. Using the following notation,

$$
\begin{aligned}
R_{m}=\sum_{i} k_{i} r_{i} W_{i}^{m} & R_{f}=\sum_{i} k_{i}\left(1-r_{i}\right) W_{i}^{f} \\
s_{m}=\sum_{i} k_{i} \delta W_{i}^{m} & s_{f}=\sum_{i} k_{i} \delta_{i} W_{i}^{f}
\end{aligned}
$$

the terms inside the brackets of (3) can be expanded in geometric series to obtain

$$
\Delta p=\frac{p}{2}\left(1+\frac{p_{11}}{p}-2 p\right)\left[\left(\frac{s_{m}}{R_{m}}-\frac{s_{f}}{R_{f}}\right)-2 p\left(\frac{s_{m}^{2}}{R_{m}^{2}}+\frac{s_{f}^{2}}{R_{f}^{2}}\right)+\cdots\right]
$$

The terms outside the brackets of $(4 a)$ are positive after one generation of random mating for $0<p<1$, so the direction of selection is given by the terms inside the brackets. If $s_{m} / R_{m} \leqq s_{f} / R_{f}$, then $A$ is selected again until lost, i.e., until $R_{m}=\bar{W}_{m}, R_{f}=\bar{W}_{f}$. If $p\left(s_{m} / R_{m}\right)$ and $p\left(s_{f} / \boldsymbol{R}_{f}\right)$ are small and $\left|s_{m} / R_{m}-s_{f} / R_{f}\right|$ is sufficiently large, then this latter value dominates the bracketed term of $(4 \mathrm{a})$, and an approximation is justified,

$$
\Delta p \simeq \frac{p}{2}(1-p)\left[\frac{s_{m}}{R_{m}}-\frac{s_{f}}{R_{f}}\right]=\frac{p}{2}(1-p)\left\{\sum_{i} k_{i} \delta_{i}\left[\frac{W_{i}^{m}}{R_{m}}-\frac{W_{i}^{f}}{R_{f}}\right]\right\},
$$

for

$$
\frac{p s_{m}}{R_{m}}, \frac{p s_{f}}{R_{f}} \ll 1, \quad p\left(\frac{s_{m}^{2}}{R_{m}^{2}}+\frac{s_{f}^{2}}{R_{f}^{2}}\right) \ll\left|\frac{s_{m}}{R_{m}}-\frac{s_{f}}{R_{f}}\right| .
$$


Expression (4b) indicates that for an allele with a small effect on sex ratio

$$
\left(p \frac{s_{m}}{R_{m}}, p \frac{s_{f}}{R_{f}} \ll 1\right) \text { and }\left|\frac{s_{m}}{R_{m}}-\frac{s_{f}}{R_{f}}\right|
$$

sufficiently large, selection acts approximately as a linear combination of its effects in different patch types. This is useful because it enables evaluation of the evolutionarily stable sex ratio by considering modifications of the sex ratio, one patch at a time. The allele may have a small effect either because it is rare $(p \ll 1)$, because the patch types it affects are infrequently encountered by zygotes $\left(k_{i} \ll 1\right)$, because the gene affects sex ratio only slightly $\left(\delta_{i} \ll 1\right)$, or any combination of these.

\section{INTERPRETATION OF THE MODEL}

From (4b), the selected sex ratio in each patch type is governed by $W_{i}^{m} / R_{m}-W_{i}^{f} / R_{f}$, approximately. To consider the implications of this, assume the simplest case of only two patch types, so that $k_{1}=k$ and $k_{2}=1-k$. Also, divide (1a) by $W_{1}^{m}$ and (1b) by $W_{1}^{f}$ and let $W_{m}=W_{2}^{m} / W_{1}^{m}$, and $W_{f}=W_{2}^{f} / W_{1}^{f}$. If allele $A$ increases the sex ratio in patch type 1 (i.e., $r_{1}^{*}>r_{1}$ ), then the direction of selection is given by

$$
\frac{1}{k r_{1}+(1-k) r_{2} W_{m}}-\frac{1}{k\left(1-r_{1}\right)+(1-k)\left(1-r_{2}\right) W_{f}}
$$

if allele $\boldsymbol{A}$ increases $r_{2}$, then selection is governed by

$$
\frac{W_{m}}{k r_{1}+(1-k) r_{2} W_{m}}-\frac{W_{f}}{k\left(1-r_{1}\right)+(1-k)\left(1-r_{2}\right) W_{f}} .
$$

These expressions indicate that selection favours evolution toward a single sex ratio equilibrium $\left(\hat{r}_{1}, \hat{r}_{2}\right)$ as a function of $k, W_{m}$, and $W_{f}$ (Table 1$)$. When the population takes on these values, invading sex ratio modifiers are selected against. These are therefore "evolutionarily stable" equilibrium sex ratios (cf. Maynard Smith, 1978), but for brevity are referred to below merely as "equilibrium" sex ratios $\left(\hat{r}_{1}, \hat{r}_{2}\right)$. The quantitative value of the sex ratio equilibrium is not immediately intuitive, but the qualitative nature of selection can be made clear. Consider $W_{m}<W_{f}$, as does Charnov (1979). This means that a patch type 2 individual relative to a patch type 1 individual is more fit as a female than as a male (even if both sexes from patch 2 are more fit than those from patch 1 ). Thus, unless the sex ratio is biased, a patch type 2 individual has higher fitness in becoming a female than a male, whereas a patch type 1 individual gains more as a male. Consequently, there is an intermediate range of $k$ for which all patch 1 individuals should become male and all patch 2 individuals female (table 1 ). If instead, one patch type is rare, then all its individuals should become the sex most benefitted, but selection favours the production of both sexes in the common patch type. Thus, at all values of $k$ (frequency of patch type 1 ), the equilibrium requires producing only one sex in at least one of the patch types $\left(\hat{r}_{1}=1\right.$, and/or $\left.\hat{r}_{2}=0\right)$.

It is interesting to contrast the case of $W_{m}<W_{f}$ to that of $W_{m}>W_{f}$ (table 1). The sex ratio equilibria are reversed, as though males in one case are 
TABLE 1

Evolutionarily stable, equilibrium sex ratios in a two-patch environment

$$
\begin{aligned}
& W_{f}, W_{m}>\frac{k}{1-k} \quad W_{f} \geqq \frac{k}{1-k} \supseteqq W_{m} \quad \frac{k}{1-k}>W_{f}, W_{m} \\
& W_{f}>W_{m} \quad \begin{cases}\hat{r}_{1}=1 & \hat{r}_{1}=1 \\
& \hat{r}_{1}=\frac{1}{2}+\frac{(1-k) W_{f}}{2 k}\end{cases} \\
& \text { (Charnov 1979) }\left\{\begin{array}{lll}
\hat{r}_{2}=\frac{1}{2}-\frac{k}{2(1-k) W_{m}} & \hat{r}_{2}=0 & \hat{r}_{2}=0
\end{array}\right. \\
& W_{f}<W_{m} \quad\left\{\begin{array}{lll}
\hat{r}_{1}=0 & \hat{r}_{1}=0 & \hat{r}_{1}=\frac{1}{2}-\frac{(1-k) W_{m}}{2 k}
\end{array}\right.
\end{aligned}
$$

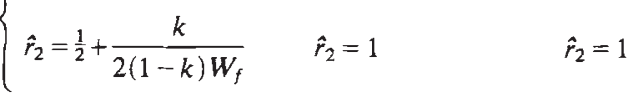

$$
\begin{aligned}
& W_{f}=W_{m}=W \quad \hat{r}_{1}=\frac{1}{2}+\frac{(1-k)\left(1-2 \hat{r}_{2}\right) W}{2 k}
\end{aligned}
$$

treated as females in the other. In the centre column of table 1 , the change from $W_{f}>W_{m}$ to $W_{f}<W_{m}$ changes the equilibrium sex ratio in patch 1 from all males to all females, and vice versa in patch 2 . Such a drastic change in sex ratio for a possibly minor change in fitness parameters is surprising. However, substitution of parameter values $W_{m} \simeq W_{f}$ into (4) shows that selection toward the equilibrium is weaker than if $W_{m}$ and $W_{f}$ differ greatly. This is important to some results below.

The assumption of two patch types may often be violated in nature. The environment may vary in a nearly continuous manner rather than consist of a few discrete patch types. It is still possible to specify the sex ratio equilibrium as a function of patch type. First, without loss of generality, order the patch types so that the ratio $W_{i}^{f} / W_{i}^{m}$ progressively increases with $i$. At the uninvadible sex ratio equilibrium, only males develop in patches with low values of this ratio (up to some value $*$ ), only females in patches with ratios greater than $*$, and possibly both sexes develop in patches whose $W_{i}^{f} / W_{i}^{m}=$ $*$. The value of $*$ depends upon the $W_{i}^{f}, W_{i}^{m}$, and $k_{i}$. In the nematode example, if male fitness is only slightly enhanced by large size but female fitness is greatly enhanced (implying that $W_{i}^{f} / W_{i}^{m}$ increases with worm size), then all worms below a certain size should become male and all above this size should become female.

The results are stated in terms of the sex ratio associated with a particular patch type. The population sex ratio is $\bar{r} \equiv \sum_{i} k_{i} \bar{r}_{i}$. It is not generally the case that the population sex ratio at equilibrium $\left(\frac{\hat{r}}{\hat{r}}\right)$ is $\frac{1}{2}$. Surprisingly, even if $W_{i}^{m}=W_{i}^{f}$ for all $i, \overline{\hat{r}}$ is not necessarily $\frac{1}{2}$, although $\overline{\hat{r}}=\frac{1}{2}$ is one of many equilibrium solutions. To see this, consider the simplest case of the twopatch model, with $k=1-k=\frac{1}{2}$, and let $W_{m}=W_{f}=W$. Equations (5) 
require that

$$
\hat{r}_{1}=\frac{1}{2}+\frac{\left(1-2 \hat{r}_{2}\right) W}{2},
$$

a line in rectangular coordinates of $\hat{r}_{1}$ and $\hat{r}_{2}$ (table 1, fig. 1). Thus, $\overline{\hat{r}}$ may vary between the extremes of $(1+W) / 4$ and $(3-W) / 4$ if $(W<1)$, or
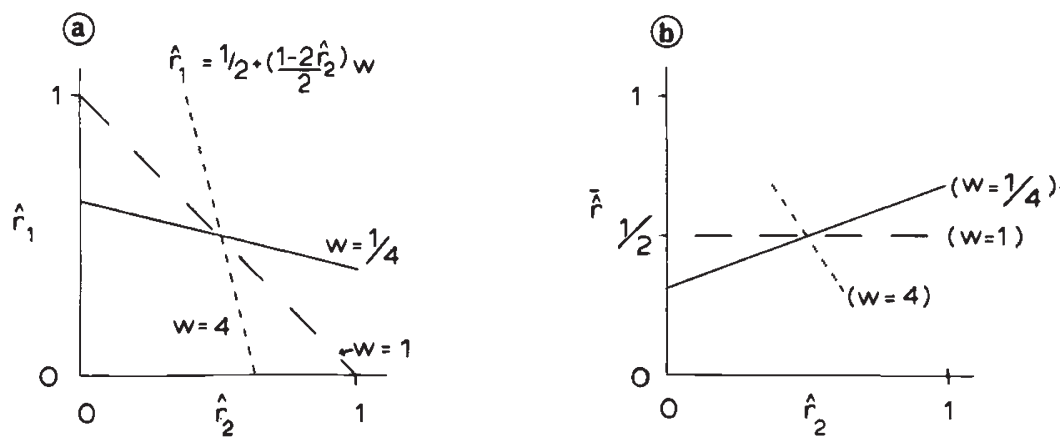

FIG. 1-The evolutionarily stable sex ratio does not require that half the zygotes become male. In a 2-patch model, $\hat{r}_{1}$ and $\hat{r}_{2}$ are the joint evolutionarily stable equilibrium proportions of males produced in patch types 1 and 2 , respectively. $W$ is the fitness of a patch type 2 individual relative to a patch type 1 individual, this being the same for males and females $\left(W_{m}=W_{f}=W\right)$. The two patch types are assumed equally common. (A) $\hat{r}_{1}$ as a function of $\hat{r}_{2}$ (as in Table 1 ), for different values of $W$. (B) The population-wide sex rato, $\overline{\hat{r}}(=$ $\left.\frac{1}{2}\left(\hat{r}_{1}+\hat{r}_{2}\right)\right)$ plotted as a function of $\hat{r}_{2} ; \bar{r}$ is $\frac{1}{2}$ at only one point.

$(W+1) / 4 W$ and $(3 W-1) / 4 W$ if $(W>1)$. This result indicates that whenever the environment is heterogeneous so as to cause differences in offspring fitness irrespective of sex, a population sex ratio of $\frac{1}{2}$ is only one of infinitely many equilibria. Yet, at all these equilibria, $\bar{W}_{m}=\bar{W}_{f}$ and the relative fitness of an individual is independent of its sex. It is shown below that this infinity of solutions depends upon a temporally constant environment, and the $\overline{\hat{r}}=\frac{1}{2}$ solution may be the only equilibrium in a fluctuating environment for $W_{m}=W_{f}$.

\section{A FLUCTUATING ENVIRONMENT}

Just as it is biologically reasonable to assume that environments are spatially heterogeneous, it is equally reasonable to assume that the environment is temporally variable, with patch type frequencies varying between generations. This means that the $k_{i}$ terms vary, and consequently, so do the $\bar{W}_{m}$ and $\bar{W}_{f}$. Results for constant-environment models do not necessarily extrapolate to fluctuating environments, and a different treatment of the model is needed.

To deal with selection in a fluctuating environment, we want an expression for the expected change in gene frequency, letting the $k_{i}$ vary between generations (with some mean $\bar{k}_{i}$ and variance $V_{k_{\mathrm{i}}}$ ). To simplify the analysis, a model with only two patch types will be considered so that only variation in $k$ need be specified $\left(k_{1}=k, k_{2}=1-k, V_{k_{1}}=V_{k_{2}}=V_{k}\right)$. From (2a) and (1), 
for allele $A$ rare, selection is approximated by

$$
\begin{gathered}
p^{(t+1)} \simeq p^{(t)} \cdot B\left[k^{(t)}\right], \\
B\left[k^{(t)}\right]=\frac{k^{(t)} r_{1}^{*}+\left[1-k^{(t)}\right] r_{2}^{*} W_{m}}{2\left\{k^{(t)} r_{1}+\left[1-k^{(t)}\right] r_{2} W_{m}\right\}}+\frac{k^{(t)}\left[1-r_{1}^{*}\right]+\left[1-k^{(t)}\right]\left[1-r_{2}^{*}\right] W_{f}}{2\left\{k^{(t)}\left[1-r_{1}\right]+\left[1-k^{(t)}\right]\left[1-r_{2}\right] W_{f}\right\}}
\end{gathered}
$$

where $k^{(t)}$ is the frequency of patch type 1 and $p^{(t)}$ is the zygotic frequency of $A$ in the th generation.

Since $B\left[k^{(t)}\right]$ depends upon time, it may exceed unity some generations but not others. Over a period of $\tau$ generations while $A$ is rare, the per-generation rate of change in $p$ is given approximately by

$$
g(k) \equiv\left\{\prod_{t=1}^{\tau} B\left[k^{(t)}\right]\right\}^{1 / \tau}
$$

where $k$ is the vector $\left[k^{(1)}, \ldots, k^{(t)}, \ldots, k^{(\tau)}\right]$.

If $g(\boldsymbol{k})$ exceeds unity, then selection favors allele $\boldsymbol{A}$. Since $g(\boldsymbol{k})$ in $(6 \mathrm{~b})$ is unique for every independent trial of $\tau$ generations, we are more interested in its expected value than its actual value. To obtain its expected value, we treat the $k^{(t)}$ as independent, identically distributed random variables from a distribution with mean $\bar{k}$ and variance $V_{k}$. Seber $(1973$, p. 7$)$ shows that

$$
E\{g(k)\} \simeq g(\bar{k})+b, \quad b=\left.\frac{V_{k}}{2} \sum_{t=1}^{\tau} \frac{\partial^{2} g(\boldsymbol{k})}{\partial\left[k^{(t)}\right]^{2}}\right|_{\boldsymbol{k}^{(t)}=\bar{k}} .
$$

This is derived by expanding $g(\boldsymbol{k})$ as a Taylor's series of $\tau$ variables about $k^{(t)}=\bar{k}$ for all $t$, and taking expected values.

Applying this to (6b), we find for $\lambda$, the approximate expected rate of change in the frequency of $A$, that

$$
\lambda \approx B(\bar{k})+\left.\frac{V_{k}}{2}\left\{\frac{\partial^{2} B(k)}{\partial k^{2}}-\frac{1}{B(\bar{k})}\left[\frac{\partial B(k)}{\partial k}\right]^{2}\right\}\right|_{k=\bar{k}} .
$$

Allele $A$ is favoured if $\lambda>1$ and is selected against if $\lambda<1$. Although not shown, the error terms truncated in deriving (7b) can be made arbitrarily small by (i) choosing a sufficiently small initial frequency of $A$, (ii) considering a sufficiently large, but bounded number of generations $(\tau)$, and (iii) choosing a distribution of $k$ with sufficiently small third and higher order moments.

In contrast to the constant-environment model, selection in a fluctuating environment no longer acts on $\boldsymbol{A}$ as a linear combination of its effects in patch 1 and 2. Results are difficult to obtain and interpret, but at least in some cases, the fluctuating- and constant-environment models give qualitatively different results, as indicated with a few special cases below.

Case I. $W_{m}=W_{f}=1$. Under these conditions all patches of the environment are equivalent in effects on fitness, and the environment is "patchy" only in effects on sex determination. The variance in sex ratio between generations is $\left(r_{1}-r_{2}\right)^{2} V_{k}$. If $r_{1}=r_{2}=\frac{1}{2}$, then the population sex ratio $(\bar{r})$ is $\frac{1}{2}$ regardless of $k$, and no sex ratio mutants can invade. Any population with some other $r_{1}$ and $r_{2}$ can be invaded, provided the mutant produces an appropriate sex ratio $\left(r_{1}^{*}, r_{2}^{*}\right)$. The population can be invaded 
even if $\bar{k} r_{1}+(1-\bar{k}) r_{2}=\frac{1}{2}$ (for $r_{1} \neq r_{2}$ ), and the few examples below lead me to conjecture that selection is acting in part to reduce the sex ratio variance.

Assuming that the mean population sex ratio through time is $\frac{1}{2}$, rare mutants of $A$ can invade under the following conditions. (a) $r_{1}^{*}=r_{2}^{*}=s$. The expected rate of increase of the rare allele is $\lambda \simeq 1+2 V_{k}\left(r_{1}-r_{2}\right)^{2}\left(1+4 s-4 s^{2}\right)$, which is maximized at $s=\frac{1}{2}$. (b) $r_{1}^{*}=r_{1}+\delta, \quad r_{2}^{*}=r_{2}$. The expected rate of change is $\lambda \cong$ $1-4 \delta V_{k}\left(r_{1}-r_{2}\right)-2 \delta^{2} V_{k}\left(1-2 r_{2}\right)^{2}$; it is sufficient that $\delta$ and $\left(r_{1}-r_{2}\right)$ have opposite signs for $A$ to increase in frequency. This formula also applies to $r_{1}^{*}=r_{1}, r_{2}^{*}=r_{2}+\delta$, if $r_{1}$ and $r_{2}$ are interchanged. A rare mutant causing $r_{1}^{*}=r_{1}+\delta, r_{2}^{*}=r_{2}+\delta$ is selected against: $\lambda \simeq 1-8 \delta^{2} V_{k}\left(r_{1}-r_{2}\right)^{2}$.

Not all mutants which invade a population will go to fixation. For example, a dominant male-determining gene $\left(r_{1}^{*}=r_{2}^{*}=1\right)$ can invade if $r_{1} \neq r_{2}$ but cannot increase to a high frequency, because it biases $\bar{r}$ toward males. However, once invaded, the male-determiner leads to a slight male-bias which in turn selects for yet additional genotypes which produce females. If the male-bias is lessened by other genotypes, the male-determiner can further increase in frequency. This process ultimately may reach a state in which $\frac{1}{2}$ of the zygotes inherit the male-determiner and the other $\frac{1}{2}$ become female (i.e., genotypic sex determination, $r_{1}=r_{2}=\frac{1}{2}$ ). Thus, although the initial change is seemingly away from a mean sex ratio of $\frac{1}{2}$, the final outcome is again a population sex ratio of $\frac{1}{2}$, and furthermore, one which is temporally constant.

Case II. $W_{m}=W_{f} \neq 1$. This is similar to case $I$ in that male and female fitness is affected equally within each patch type, but differs from I in that one patch type is more advantageous to fitness than the other. If the frequency of patch types is constant from generation to generation, then selection favors

$$
\hat{r}_{1}=\frac{1}{2}+\frac{(1-k)\left(1-2 \hat{r}_{2}\right) W}{2 k} \quad(\text { table } 1)
$$

This sex ratio can be invaded in a fluctuating environment, however, unless $r_{1}=r_{2}=\frac{1}{2}$. A rare allele causing $r_{1}^{*}=r_{2}^{*}=\frac{1}{2}$ can invade, and so can at least one of $r_{1}^{*}=r_{2}^{*}=0,1$. Possibly, therefore, $\hat{r}_{1}=\hat{r}_{2}=\frac{1}{2}$ is the unique equilibrium for $W_{m}=W_{f}$.

Case III. $W_{m}<W_{f}$. One of the more striking aspects of sex ratio evolution in spatially heterogeneous environments with constant $k_{i}$ is that selection favours producing all males in some patches and all females in other patches (see above, table 1). The two cases above (for $W_{m}=W_{f}$ ) caution the extrapolation of results from constant-environment models to fluctuating-environment models, but there are parameter values for which a sex ratio $(1,0)$ is uninvadible in a fluctuating as well as a constant environment. Table I shows that $\hat{r}_{1}=1, \hat{r}_{2}=0$ for $W_{m} \leqq k /(1-k) \leqq W_{f}$. In a fluctuating environment, the sex ratio $(1,0)$ is stable against invasion provided

$$
W_{m} \leqq \frac{\bar{k}}{1-\bar{k}+\left(V_{k} / \bar{k}^{2}\right)} \quad W_{f} \geqq \frac{\bar{k}}{1-\bar{k}}+\frac{V_{k}}{(1-\bar{k})^{3}} .
$$

The variance in $k$ merely decreases the zone in which $(1,0)$ is stable. If $W_{m}$ is only slightly less than $W_{f}$, then $(1,0)$ may not be stable under any 
circumstances (for $V_{k}$ sufficiently large). This is not surprising in view of the weakness of selection toward this equilibrium in a constant environment when $W_{m} \simeq W_{f}$ (cf. equations (4), (5)).

In comparison to sex ratio selection in a constant environment, sex ratio selection in a fluctuating environment tends to favour production of both sexes within a patch. This tendency is overcome if $W_{m}$ and $W_{f}$ are sufficiently different and $\bar{k}$ is in the appropriate range.

\section{ChOICE OF PATCH TYPE}

In addition to genetic variation for sex ratio, there may be genetic variation for assortment to patch type. Mothers may be able to recognize and to choose nesting sites according to effects on offspring fitness. In Bonellia, a marine worm with environmental sex determination, larvae settle on the ocean floor to become adults. Those settling on adult females become male, while those larvae settling on uninhabited substrate become females (Bacci, 1965; Leutert, 1975). Possibly, a Bonellia larva can exhibit a choice of patch type by refusing to settle on a female. Choice may not always be possible or may involve a cost, but interest here is restricted to the simplest case, that in which choice is cost-free.

The evolution of patch choice can be studied in the same way as sex ratio, by allowing the allele $A$ to produce a $k_{i}^{*}$ rather than $k_{i}$ (holding sex ratio constant). In the two-patch model, selection on a rare allele causing $k_{i}^{*}=k_{i}+\delta$ in heterozygotes is governed approximately by

$$
\frac{\delta}{2}\left[\frac{r_{1}-r_{2} W_{m}}{k r_{1}+(1-k) r_{2} W_{m}}+\frac{\left(1-r_{1}\right)-\left(1-r_{2}\right) W_{f}}{k\left(1-r_{1}\right)+(1-k)\left(1-r_{2}\right) W_{f}}\right] .
$$

If $W_{m}<W_{f}$ and $r_{1}$ and $r_{2}$ are at equilibrium values (table 1), there are three uninvadible equilibria for $k$. (1) $\hat{k}=\frac{1}{2}$ if $W_{m}<1<W_{f}$. (2) $\hat{k}=1$ if $W_{m}, W_{f}<$ 1. (3) $\hat{k}=0$ if $W_{m}, W_{f}>1$. These equilibria are quite reasonable. If male and female fitnesses are both greater in one patch type than in the other, selection favours all zygotes going to the patch of high fitness (assuming no density-dependent fitness components). If males are more fit in one patch type but females more fit in the other, then selection favours males assorting to the one and females to the other in equal numbers. In all cases the joint equilibrium of $\hat{k}, \hat{r}_{1}, \hat{r}_{2}$ produces a population sex ratio $(\overline{\hat{r}})$ of $\frac{1}{2}$.

\section{EVOLUTION OF THE ENVIRONMENTAL CUE CONTROLLING SEX RATIO}

Why does the sex of mermithid nematodes depend upon crowdedness of the host, the sex of Bonellia depend upon the presence or absence of an adult female, and the mother wasp control her offspring's sex in response to host size? It would be difficult to argue convincingly that there is some common physiological basis for the sex determining cue in these various species. Instead, it may be that these cues have underlying selective similarities. The cue seems to be highly correlated with environmental circumstances in which there is a major difference in fitness between becoming a male or a female. In mermithids and wasps, adult size may be highly correlated with fitness as a female, but less correlated with fitness as a male (see Discussion). To a juvenile worm undergoing sex differentiation, 
host crowdedness may be a good indicator of its expected adult size; to a mother wasp, host size may be a good indicator of how big her offspring will be. In Bonellia, whether a larva settles in the open or on an adult female, makes a big difference to its fitness as a male or a female. The essential feature of these cues is that they indicate a differential benefit to maleness versus femaleness, not merely indicating the benefit to one sex independently of the other.

As a general problem, it is difficult to understand the evolution of the cue used in sex ratio control, because the change from one environmental cue to another may involve changes in all parameters, $k_{1}, r_{i}, W_{i}^{m}$, and $W_{i}^{f}$, yet only the $W$ 's are of interest. The formula for change in the cue is given by (2), where the $k_{i}, r_{i}, W_{i}^{m}$, and $W_{i}^{f}$ associated with allele $A$ are different from those of $a$, but no simple conclusion is apparent from this formula. Instead of pursuing a general formulation, then we pursue a specific one. Consider an environmental parameter $x$ which ranges from 0 to 1 , and let male and female fitnesses be linear functions of the value $x$ of the patch type in which they are raised (fig. 2). Recalling the arguments given above, the uninvadible sex ratio in this case is all males for values of $x$ less than some value (*) and all females for $x$ greater than (*) (fig. 2). To simplify the calculations, further assume that (i) the patch type values are regularly and densely spaced in $0<x<1$, and (ii) all patch types are encountered with equal probability. Thus we may approximate $\bar{W}_{m}\left(\bar{W}_{f}\right)$ as the area under the male-fitness

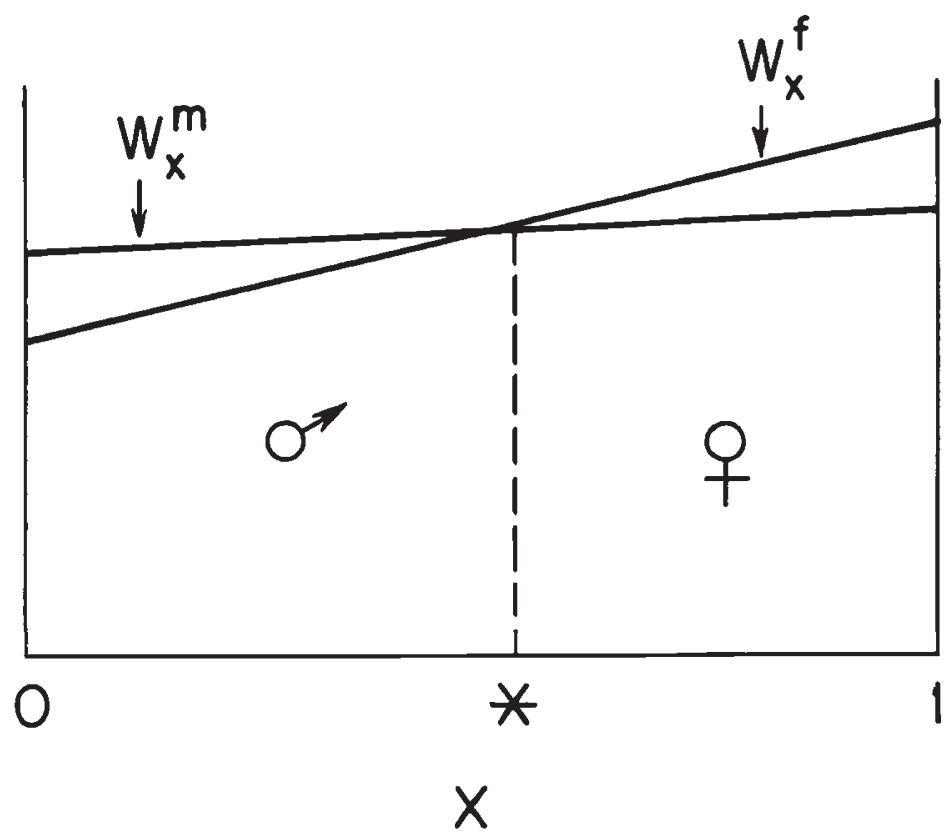

FIG. 2.-Hypothetical relationship between male fitness $\left(W_{x}^{m}\right)$ and female fitness $\left(W_{x}^{f}\right)$ as a function of patch type $(x), x$ is assumed to take on many (but a finite number of) values between 0 and 1 , and is represented by a continuous variable. $(*)$ is the threshold of sex determination, with males developing for all values of $0 \leqq x<*$ and females developing for all values of $*<x \leqq 1$. As discussed in the text, the question is posed as to whether selection favors sex determination in response to $x$ or some other cue $(y)$, which has different slopes of its $W_{y}^{m}$ and $W_{y}^{f}$ functions. 
(female-fitness) line for $0<x<*(*<x<1)$. With this formulation, we can ask if selection will favour embryos controlling sex ratio in response to some other environmental cue $(y)$, which has different associated linear fitness functions (but is otherwise similar to $x$ in its simplifying assumptions). The analysis (unpublished) shows that in order for a new cue to evolve, the difference between the slopes of the female and male fitness functions for $y$ must at least exceed the difference in slopes for $x$. That is, the new cue must be better at differentially indicating benefits to maleness versus femaleness (for each $i,\left|W_{i}^{f}-W_{i}^{m}\right|$ must increase). The evolution of the new cue is also constrained by the sex ratio it produces, but this is not a complication if an intermediate value of $y$ (which corresponds roughly to $*$ ) is used as a threshold of sex determination. The conclusion from this analysis is therefore consistent with the observation that the environmental cue used in sex ratio control is one which indicates a differential benefit to maleness versus femaleness.

\section{Discussion}

This paper investigates the evolution of the sex ratio in spatially heterogeneous (patchy) environments, assuming random mating among all patches in the environment. The models differ from most previous treatments of sex ratio evolution in that offspring reared in different patches of the environment have correspondingly different fitnesses as adults. If there is a differential benefit to males versus females in different environmental patches, then selection favours over-producing the sex which is most benefitted by the patch, as recognized by Trivers and Willard (1973), Charnov and Bull (1977), Charnov et al. 1978, and Charnov (1979). For example, if an individual can become an above-average male or an average female, selection favours becoming the male, provided the population sex ratio is not already heavily male-biased. The selected sex ratios associated with the different patches are often as extreme as all-male or all-female, some patches in the population producing males and other patches producing females.

The observations on some species are seemingly in agreement with the qualitative predictions of the models. Parasitic wasps tend to put males in small hosts, females in large hosts, though the evidence that females benefit more than males by large size is only circumstantial (Charnov, 1979; Charnov et al., 1981). Mermithid nematodes become males upon crowding, become female when not crowded and when the host is well-fed (Christie, 1929; Bacci, 1965; Harlos et al., 1980). There are no data on fitness of males and females of different sizes, but it seems likely that females benefit more from large size, because the male is usually the smaller sex in namatodes regardless of the sex determining mechanism (Chitwood and Chitwood, 1974). Bonellia larvae which settle on an adult female enter her reproductive tract and develop into dwarf males; larvae which settle in open substrate become females (Leutert, 1975). There is little doubt that intrauterine existence benefits maleness more than femaleness. Bonellia does not strictly apply to the above analysis, because patch types are sex ratio dependent, but a slightly modified analysis gives nearly the same result. Other examples are discussed in this context by Charnov and Bull (1977).

An example which has recently come to light is that incubation temperature controls sex determination in various reptiles (reviewed by Bull, 1980). This mechanism is apparently widespread in turtles, and also occurs in 
lizards and alligators. In most turtles studied, low temperatures produce males and high temperatures females, but the snapping turtle (and perhaps others) become female at extreme low temperatures also. Lizards, however, show the reverse of most turtles: males develop at high temperatures, females at low temperatures. As yet, there is no reason to suppose that incubation temperature differentially affects fitness in males and females. The hatchling turtle may require many years to mature and it increases in mass several hundredfold, so it is not clear that there are residual effects extending from hatching to adulthood. Furthermore, it must be argued that the relative advantage of incubation temperature to male versus female fitness is the reverse in turtles as in lizards. An alternative, of course, is that temperature is irrelevant to subsequent fitness as a male or female, but instead allows the mother to control the sex of her offspring. In this case, fitness as male or female is expected to correlate with next temperature, even though the next environment is not the factor influencing fitness. There is no evidence on ths possibility either.

The models assume that (1) the offspring has the ability to control its sex (or, in (2c), that a parent can control offspring sex ratio), and (2) sex ratio control is in response to some environmental stimulus which correlates with fitness. In considering the relevance of these models to a broad range of species, it may be that the first requirement, sex ratio control, is quite restrictive. The species discussed above have either environmental sex determination or haplo-diploidy. In species with environmental sex determination, the embryo is clearly able to determine its sex, and in haplo-diploids the mother can control offspring sex ratio by differential fertilization of eggs (Flanders, 1965). However, a majority of animals have the less flexible mechanisms of male and female heterogamety (XY/XX). Sex ratio control may be difficult with these mechanisms because it requires either modifying the segregation ratio of the $X$ and $Y$ or differentially controlling fertilization of eggs by $X$ - and $Y$-sperm. The inflexibility of genotypic sex determination to sex ratio modification was the basis for Charnov and Bull's (1977) hypothesis concerning the evolution of environmental sex determination. If some patch type favours an embryo to become male (for example), genotypic sex determination will also unavoidably produce some females in the patch, even though these females are of relatively low fitness. Selection might favour embryos which overcome the genotypic control and determine sex in response to the environment, because these embryos always avoid developing into the sex of low fitness.

It seems a priori unlikely that species with male or female heterogamety are able to control sex ratio to the extent of conceiving a son versus a daughter at will, but some level of control is plausible. Williams (1979) analyzed family sex ratios in some non-domestic vertebrates and concluded that all evidence was consistent with binomial variance, as expected from Mendelian segregation of a pair of alleles or chromosomes. Certain effects are known in laboratory mice, however, both in which the mean sex ratio differs from $\frac{1}{2}$ and in which the sex ratio differs between first and subsequent litters (Weir, 1962). It is not clear if these biases reflect biases in the conceived sex ratio, or a subsequent differential loss of male and female embryos. Howe (1977) reports what may be short-term seasonal effects in the primary sex ratio of a bird. Maternal age and environmental temperature affect sex ratios in two insects, a moth and a scale (Seiler, 1920; Nelson-Rees, 1960). In at least the moth, temperature has been shown to 
differentially affect the segregation of sex chromosomes to the polar body of the egg. Thus, certain types of sex ratio responses might be selected in species with male and female heterogamety.

The ability to recognize an environmental cue which differentially correlates with male/female fitness is another requirement for the evolution of sex ratio modification. In species with environmental sex determination, this must often be a physiological mechanism whereby the embryo's sex responds to some chemical indicator, as in mermithid larvae in a host, or Bonellia larvae settling on a female. In hymenopteran parasitoids, the mother can apparently assess host size, which is an appropriate cue for sex ratio control (Charnov, 1979; Charnov et al., 1981). Trivers and Willard (1973) suppose that the physiology of a pregnant mammal may vary depending upon her nutritional condition, and that the sex ratio response could evolve in conjunction with some physiological indicator (but there is yet little evidence of such facultative sex ratio responses in mammals, Myers, 1978).

The models consider the selected sex ratio associated with particular patch types. The average sex ratio in the population at the evolutionarily stable equilibrium is not generally $\frac{1}{2}$. To take an extreme case, if $\frac{3}{4}$ of the zygotes encounter situations in which male embryos die but female embryos are unaffected, then selection favors this $\frac{3}{4}$ of the zygotes to become female despite any population-wide sex ratio bias: as females they have average fitness, but as males they have zero fitness. A more surprising result is that the population sex ratio may be biased at equilibrium even if there are no male/female differences associated with patch type. If some parts of the environment differentially enhance zygote survival or fertility independently of sex, then there are an infinite number of sex ratio equilibria at which the population sex ratio is not $\frac{1}{2}$. This result is also made clear with an example. If half the eggs enter an environment which causes them to be sterile, then their sex is irrelevant to selection. They may be all males or all females, but selection will favour a sex ratio of $\frac{1}{2}$ in the remainder of the environment (table 1). The total population sex ratio could be as extreme as $\frac{1}{4}$ or $\frac{3}{4}$. The same principle applies when there are less extreme differences in fitness. At all equilibria the relative fitness of an individual is independent of its sex. (This is not usually true of equilibria when male/female fitnesses differ within a patch.)

At first sight, these results seem to contradict Fisher's claim (1930) that "the total parental expenditure incurred in respect of children of each sex, shall be equal". Fisher's meaning of "equal expenditure" is not clear, however. If it is interpreted to be that equal resources are converted into male as into female zygotes (with no post-conception parental care), then "equal expenditure" is not necessary at equilibrium whenever sex ratios correlate with an environmental parameter that correlates with fitness (viability or fertility).

Sex ratio selection is affected by changes in the frequencies of patch types through time, i.e., a fluctuating environment. If there are no sex differences in fitness within patches, then the infinity of sex ratio equilibria observed in constant-environment models is reduced to possibly one-a sex ratio of $\frac{1}{2}$ in all patches. This corresponds to "equal expenditure" on zygotes of the two sexes. If males and females differentially benefit with patch type, then a fluctuating environment tends to favour producing both sexes within a patch, but this favoured tendency is completely overcome if there is a 
sufficient difference in male/female fitness. Thus, in nature, one expects to observe all-males or all-females associated with particular environmental circumstances if and only if there is a marked differential benefit according to sex. It is for this reason that the occurrence of environmental sex determination in reptiles is puzzling.

To conclude, the theory presented here for the evolution of sex ratios in a spatially heterogeneous environment corroborates the major predictiuns of Charnov (1979) regarding the evolutionarily stable equilibria. It goes further in

(i) Generalizing a single-locus model of the evolutionary process.

(ii) Considering the evolution of parameters other than the sex ratio.

(iii) Introducing the implications of fluctuating environments. There are yet weakness of this theory that warrant improvement.

The control of sex ratio in species with environmental sex determination or haplo-diploidy is likely polygenic, and quantitative genetic models of sex ratio evolution are preferred to the single locus treatments. The values of the predicted equilibria are not likely to differ under polygenic inheritance, but the dynamical approach to these equilibria will be affected. Further development of this theory is warranted, because these sex ratio phenomena are amenable to laboratory and field study in various species.

Acknowledgements. - This paper was inspired by several years of interaction with E. L. Charnov. I thank him, B. and D. Charlesworth, K. Aoki, and M. Moody for reading and commenting upon the manuscript and R. Lande for discussion. W. Engels introduced me to the method for studying selection in fluctuating environments. I was supported by an NIH Postdoctoral Fellowship (Madison) and a NATO Fellowship (Sussex) during the preparation of this paper. This is paper \# 2451 from the Laboratory of Genetics, U.W. Madison.

\section{REFERENCES}

BACCI, G. 1965. Sex Determination. Pergamon Press, Oxford.

BUll, J. J. 1980. Sex determination in reptiles. Quart. Rev. Biol. 55, 3-21.

CHARNOV, E. L. 1979. The genetical evolution of patterns of sexuality: Darwinian fitness. Am. Nat., 113, 465-480.

CHARNOV, E. L., AND BULL, J. J. 1977. When is sex environmentally determined? Nature, $266,828-830$.

CHARNOV, E. L., GOTSHALL, D., AND ROBINSON, J. 1978. Sex ratio: adaptive response to population fluctuations in pandalid shrimp. Science, 200, 204-206.

CHARNOV, E. L., LOS-DEN HARTOGH, R. L., JONES, T., AND VAN DEM ASSEM, J. (in preparation). Sex ratio evolution in a patchy environment.

CHITwOOD, B. G., AND CHITwOOD, M. G. 1974. An introduction to Nematology (revised). University Park Press, Baltimore.

CHRISTIE, J. R. 1929. Some observations on sex in the mermithidae. J. Exp. Zool., 53, 59-76.

FISHER, R. A. 1930. The Genetical Theory of Natural Selection, (reprint 1958). Dover, New York

FLANDERS, S. E. 1965. On the sexuality and sex ratios of hymenopterous populations. Am. Nat., 99, 489-494.

HAMILTON, W. D. 1967. Extraordinary sex ratios. Science, 156, 477-488.

HARLOS, J., BRUST, R. A., AND GALLOWAY, T. D. 1980. Observations on a nematode parasite of Aedes vexans (Diptera:Culicidae) in Manitoba. Can. J. Zool., 58, 215-220.

HOWE, H. 1977. Sex-ratio adjustment in the common grackle. Science, 198, 744-746.

KIDWELL, J. F., CLEGG, M. T., STEWART, F. M., AND PROUT, T. 1977. Regions of stable equilibria for models of differential selection in the two sexes under random mating. Genetics, 85, 171-183.

LEUTERT, R. 1975. Sex-determination in Bonellia. In Intersexuality in the Animal Kingdom, ed. R. Reinboth. Springer-Verlag, Berlin.

MAYNARD SMITH, J. 1978. The Evolution of Sex. Cambridge University Press, Cambridge. MYERS, J. H. 1978. Sex ratio adjustment under food stress: maximization of quality or numbers of offspring? Am. Nat., 112, 381-388. 
NELSON-REES, W. A. 1960. A study of sex predetermination in the mealy bug Planococcus citri (Risso). J. Exp. Zool., 144, 111-137.

SEBER, G. A. F. 1973. The Estimation of Animal Abundance and Related Parameters. Griffin, London.

SEILER, J. 1920. Geschlechtschromosomenuntersuchungen an Psychiden. I. Experimentelle Beinflussung der Geschlechts bestimenden Reifeteilung bei Talaeporia tubulosa. Retz. Arch. Zellforsch., 15, 249-268.

SHAW, R. F. 1958. The theoretical genetics of the sex ratio. Genetics, 43, 149-163.

SHAW, R. F., AND MOHLER, J. D. 1953. The selective significance of the sex ratio. Am. Nat., $87,337-342$.

TRIVERS, R. L., AND WILLARD, D. E. 1973. Natural selection of parental ability to vary the sex ratio of offspring. Science, 179, 90-92.

WEIR, J. A. 1962. Hereditary and environmental influences on the sex ratio of PHH and PHL mice. Genetics, 47, 881-897.

WILliamS, G. C. 1979 . The question of adaptive sex ratio in outcrossed vertebrates. Proc. $R$. Soc. Lond. B, 205, 567-580.

WRIGHT, S. 1969. Evolution and the Genetics of Populations, Vol. 2. University of Chicago Press, Chicago.

WRIGHT, S. AND DOBZHANSKY, T. 1946. Genetics of natural populations. XII. Experimental reproduction of some of the changes caused by natural selection in certain populations of Drosophila pseudoobscura. Genetics, 31,125-156.

Notation

\section{APPENDIX}

$A, a$ two alleles with different effects on the sex ratio

an evolutionarily stable equilibrium value; superscript

$k_{i} \quad$ frequency with which zygotes encounter patch type $i$

$k$ frequency of patch type 1 (2-patch model only)

$p_{11}, 2 p_{12}, p_{22} \quad$ zygotic frequency of genotype $A A, A a, a a$, respectively

$p \quad$ zygotic frequency of allele $A\left(=p_{11}+p_{12}\right)$

$r_{i}^{* *}, r_{i}^{*}, r_{i}$ probability that genotype $A A, A a$, aa, respectively, becomes male in patch type $i$

$\delta_{i} \quad$ the additive effect of allele $A$ on sex ratio in patch type $i$ $\left(r_{i}^{* *}=r_{i}+2 \delta_{i}, r_{i}^{*}=r_{i}+\delta_{i}\right)$

$\bar{r}_{i}$ average sex ratio in patch $i$ prior to fitness effects $\left(=p_{11} r_{i}^{* *}+2 p_{12} r_{i}^{*}+p_{22} r_{i}\right)$

$\bar{r}$ average population sex ratio prior to fitness effects $\left(=\sum_{i} k_{i} \bar{r}_{i}\right)$

$W_{i}^{f}, W_{i}^{m}$ fitness of a female, male, respectively, reared in patch type $i$

$W_{f}, W_{m}$ relative fitness of a female, male, reared in patch type 2 $\left(=W_{2}^{f} / W_{1}^{f},=W_{2}^{m} / W_{1}^{m}\right)$, respectively (2-patch model only)

$\stackrel{*}{W}, \stackrel{*}{W}$,

marginal fitness of allele $A$ in females $\left\{=\left(\sum_{i} k_{i}\left[p_{11}(1-\right.\right.\right.$ $\left.\left.\left.\left.r_{i}^{* *}\right)+p_{12}\left(1-r_{i}^{*}\right)\right] W_{i}^{f}\right) / p\right\}, \quad$ males $\quad\left[=\left(\sum_{i} k_{i}\left(p_{11} r_{i}^{* *}+\right.\right.\right.$ $\left.\left.\left.p_{12} r_{i}^{*}\right) W_{i}^{m}\right) / p\right]$, respectively

$\bar{W}_{f}, \bar{W}_{m}$ population average of female, male, fitness times their proportion in the patch $\left[=\sum_{i} k_{i}\left(1-\bar{r}_{i}\right) W_{i}^{f},=\sum_{i} k_{i} \bar{r}_{i} W_{i}^{m}\right]$, respectively

$R_{f}, R_{m} \quad \bar{W}_{f}, \bar{W}_{m}$ in the absence of allele $A\left[=\sum_{i} k_{i}\left(1-r_{i}\right) W_{i}^{f}\right.$, $=\sum_{i} k_{i} r_{i} W_{i}^{m}$, respectively

$s_{f}$ minus the contribution that allele $A$ makes to females $\left(=\sum_{i} k_{i} \delta_{i} W_{i}^{f}\right)$ (additive effect only)

$\mathrm{s}_{\mathrm{m}}$ the contribution that allele $A$ makes to males $\left(=\sum_{i} k_{i} \delta_{i} W_{i}^{m}\right)$ (additive effect only)

$V_{k}$ temporal variance in the frequency of patch type 1 (2-patch model) 